\title{
5 Mills deontische Konkretisierung des Freiheitsprinzips ${ }^{1}$
}

\subsection{Schädigung als Verletzung dreier Typen von Pflichten}

In Kapitel IV schickt Mill sich an, die Hauptfrage von Über die Freiheit nach der inhaltlichen Grenze legitimer gesellschaftlicher Macht abschließend zu beantworten: „Worin also bestehen die rechtmäßigen Grenzen der Souveränität des Individuums über sich selbst? Wo beginnt die Autorität der Gesellschaft?“ (CW XVIII, S. 276). ${ }^{2}$ Mit anderen Worten: Wo endet das moralische Recht des Individuums auf freie Selbstkultivierung? ${ }^{3}$ Wo beginnt das moralische Kontrollrecht ${ }^{4}$ der Gesellschaft, dem Einzelnen Pflichten aufzuerlegen und Gehorsam zu erzwingen? Die zweite Frage erinnert daran, dass Mills Freiheitsprinzip „den Umgang der Gesellschaft mit dem Individuum durch Zwang und Kontrolle [...] absolut zu regeln“ beansprucht (CW XVIII, S. 223; meine Hervorh.); und dass Gesellschaften

1 Eine erster Entwurf dieses Textes wurde im Mai 2013 auf einem Mill-Workshop an der Universität Lüneburg präsentiert, zu dem Michael Schefczyk eingeladen hatte. Eine zweite Fassung wurde im April 2014 auf dem Berner Forschungskolloquium für Praktische Philosophie diskutiert. Den Teilnehmern beider Diskussionsrunden möchte ich dafür herzlich danken. Besonderen Dank für besonders detaillierte Kommentare und Anmerkungen schulde ich Claus Beisbart, Michael Schefczyk und Thomas Schramme.

2 Alle Übersetzungen aus dem Englischen stammen von mir.

3 In seiner Autobiografie bezeichnet Mill Über die Freiheit rückblickend als „philosophisches Lehrbuch einer einzigen Wahrheit [...]: die Bedeutung, für Mensch und Gesellschaft, einer großen Vielfalt an Charaktertypen und des Einräumens voller Freiheit der menschlichen Natur, sich in zahllose und konfligierende Richtungen zu entfalten“ (Mill 1873, S. 259). Das, so Mill an gleicher Stelle, sei die Hauptpointe seiner „Lehre von den Rechten [rights] der Individualität und des Anspruchs der moralischen Natur [des Menschen], sich auf eigene Weise zu entwickeln“ (Mill 1873, S. 260). Denselben Punkt bringt das Motto von Über die Freiheit zum Ausdruck.

4 Für Mill besteht die Autorität einer Gesellschaft in einem moralischen Recht, ihre Mitglieder durch Auferlegung und Durchsetzung von Pflichten zu kontrollieren. Dieses gesellschaftliche Kontrollrecht - „the right to control“ CW XVIII, S. 279-283 - zerfällt in eine Befugnis, Pflichten aufzuerlegen und in eine Erlaubnis, sie auszuüben und die resultierenden Pflichten gegebenenfalls mit Zwang durchzusetzen. Diese gesellschaftliche Befugnis ist das moralische Gegenstück zu einer Hohfeldschen „power“, der seitens des Bürgers eine (erneut moralisch zu verstehende) Hohfeldsche „liability“ - Mill spricht von „amenability“ (CW XVIII, S. 223-224) korrespondiert. Vgl. Hohfeld 1913. 
autoritativen Zwang und autoritative Kontrolle durch Auferlegung und Durchsetzung positiv-rechtlicher und positiv-moralischer Regeln ausüben. Wir können Mills Kernfrage daher äquivalent, aber präziser so reformulieren: Welche verpflichtenden Zwangsregeln darf eine Gesellschaft ihren Mitgliedern moralisch gerechtfertigt aufbürden? Oder auch: Welche positiven Pflichten darf sie für ihre Bürger gerechtfertigt setzen? In seiner Antwort in Kapitel IV präzisiert und differenziert Mill den in seiner ersten, noch provisorischen Formulierung des Freiheitsprinzips in Kapitel I (CW XVIII, S. 223) verwendeten Begriff der Schädigung [harm] durch die Unterscheidung dreier Typen gesellschaftlicher Pflichten [social obligations] und ihrer Verletzung.

Um welche Typen gesellschaftlicher Pflichten geht es? Betrachten wir sie der Reihe nach. Als erstes nennt Mill die grundlegende Pflicht jedes Bürgers, „die Interessen anderer nicht zu verletzen; oder vielmehr gewisse Interessen, die, entweder durch eine explizite rechtliche Vorschrift oder durch stilles Einverständnis, als Rechte betrachtet werden sollten“ (CW XVIII, S. 276). Wohlgemerkt: Nicht alle, sondern nur „gewisse Interessen“ sollten als Rechte betrachtet werden. An welche Unterklasse menschlicher Interessen denkt Mill hier? Offenbar vor allem an jene „Nützlichkeiten im weitesten Sinne“, die „in den dauerhaften Interessen des Menschen als eines fortschrittlichen Wesens“ gründen (CW XVIII, S. 224). Gesellschaften, so Mill, sind die „Beschützer all ihrer Mitglieder“ (CW XVIII, S. 280). Sie sollen die Interessen ihrer Mitglieder schützen. Das ist ihre Primärfunktion, und wenn es um die „dauerhafte“ und „vitale“ Interessen der Bürger geht, wird dieses Sollen zu einem „Müssen“, d. h. zu einer gesellschaftlichen Schutzpflicht (CW X, S. 251). Das ist die Pointe der oben zitierten Bemerkung Mills, dass die Gesellschaft - kollektiv und individuell - „gewisse Interessen“ des Einzelnen durch „explizite rechtliche Vorschrift“ oder „stillschweigendes Einverständnis“ als zwangsbewehrte Rechte anzuerkennen habe. Die Gesellschaft manifestiert ihre Anerkennung bestimmter vitaler Interessen als moralische Rechte, indem sie ihren Mitgliedern korrelative positiv-rechtliche oder positiv-moralische Schutzpflichten auferlegt. ${ }^{5}$ Da die positiven Pflichten dieses ersten Pflichttyps dem

5 Warum sollte, ja, muss die Gesellschaft bestimmte moralischen Rechte ihrer Mitglieder anerkennen und positivieren? Mills Antwort ist klar: „Wenn ein Kritiker fragt, warum sie es sollte, kann ich ihm keinen anderen Grund geben als allgemeine Nützlichkeit“ (CW X, S. 250). Dass die moralischen Rechte des Einzelnen in allen Gesellschaften positiv-rechtlich anerkannt und de jure durchgesetzt werden sollten, ist jedoch logisch verträglich damit, dass sie de facto nirgends anerkannt oder durchgesetzt werden. Die Existenz der zu positivierenden moralischen Rechte ist von ihrer gesellschaftlichen Anerkennung und faktischen Positivierung unabhängig. Denn ihr Besitz - und somit ihre Existenz - folgt aus einer „gewissen Klasse moralischer Regeln, welche die Notwendigkeiten [essentials] menschlichen Wohls näher betreffen“ (CW X, S. 255). Sie folgt 
Schutz vitaler, ,in den dauerhaften Interessen des Menschen als eines fortschrittlichen Wesens“ (CW XVIII, S. 224) fundierter Interessen dienen, liegt es nahe, sie als „vitale Schutzpflichten“ zu bezeichnen.

Welchen Inhalt haben vitale Schutzpflichten des ersten Pflichttyps? Sie fordern mehrheitlich (passive) Unterlassungen: nicht $\mathrm{zu}$ morden, nicht $\mathrm{zu}$ stehlen, nicht zu betrügen, etc. Mill erkennt jedoch auch vitale Schutzpflichten an, die ihren Trägern (aktive) Akte der Begünstigung abverlangen. Denn, so Mill, eine „Person kann anderen nicht nur durch ihr Tun, sondern auch durch ihre Untätigkeit Übel verursachen und in beiden Fällen ist sie ihnen für die Verletzung zu Recht verantwortlich“ (CW XVIII, S. 225). Mill denkt hier an „gewisse Akte individueller Begünstigung, wie die Rettung des Lebens eines Mitmenschen oder das Dazwischengehen zur Verteidigung der Wehrlosen gegen Missbrauch“ (CW XVIII, S. 225). Aktive Begünstigungen dieser Art sind nicht supererogatorisch, sondern erogatorisch, weil sie direkt aus einem moralischen Recht des Opfers folgen. ${ }^{6}$ Halten wir fest, dass nicht nur Unterlassungen, sondern auch Begünstigungen für Mill zu den vitalen Schutzpflichten des ersten Pflichttyps gehören. Bei beiden handelt es sich um vollkommene Pflichten der Gerechtigkeit, denen ,kraft derer sich in einer oder mehreren Personen ein korrelatives Recht [a correlative right] befindet“ (CW X, S. 247).

Auch die Instanziierungen des zweiten von Mill genannten Pflichttyps sind vollkommene Pflichten der Gerechtigkeit. Auch sie dienen letztlich dem Schutz vitaler Interessen. Allerdings auf indirekte Weise, insofern sie sich aus institutionellen Notwendigkeiten ihrer direkten Sicherung ergeben. Denn ein wirksamer, effektiver Schutz vitaler Interessen, so Mill, erfordert eine institutionelle „Maschinerie zu ihrer Bereitstellung“ (CW X, S. 251). Die Institutionalisierung dieser „Maschinerie“ und ihr reibungsloses Arbeiten habe jedoch ihre Kosten: Sie Sei mit „Mühen und Opfern“ verbunden, die eine Gesellschaft durch Auferlegung vollkommener Pflichten auf ihre Mitglieder umlegen darf. Die Gesellschaft könne verlangen, „dass jede Person ihren (durch einen Billigkeitsgrundsatz zu bestimmenden) Beitrag leistet zu den Mühen und Opfern, die bei der Verteidigung der Gesellschaft und ihrer Mitglieder gegen Verletzung und Belästigung anfallen“ (CW XVIII, S. 276). Als Beispiele institutioneller Pflichten des Bürgers „zum Wohl anderer, zu deren Erfüllung er berechtigt gezwungen werden darf,“ nennt Mill:

aus den Regeln der Gerechtigkeit, die für Mill dadurch definiert sind, dass sie individuellen Rechte generieren (CW X, S. 255).

6 Das heißt natürlich nicht, dass Mill die Existenz supererogatorischer Handlungen bestreiten würde: „Es gibt ein Maß an Altruismus, das allen abverlangt werden sollte, und eine Stufe, über die hinaus er nicht obligatorisch ist, aber verdienstvoll“ (Mill 1865a, S. 337; meine Hervorh.). 
Vor „Gericht als Zeuge auszusagen; seinen fairen Anteil an der gemeinsamen Landesverteidigung zu tragen; oder an irgendeinem anderen gemeinschaftlichen Vorhaben im Interesse der Gesellschaft, deren Schutz er genießt“ (CW XVIII, S. 225). Mills wiederholter Hinweis auf die Rolle der Gesellschaft als „Beschützer all ihrer Mitglieder“ macht klar, dass es sich auch hier durchweg um Pflichten handelt, deren Erfüllung letztlich der Sicherung vitaler Interessen dient. Auch sie sind vollkommene Pflichten, auf deren pünktliche Erfüllung jeder, der seinen fairen Anteil zu Aufrechterhaltung der Schutzinstitutionen leistet, gegenüber allen ein Recht hat, die von ihrem Schutz profitieren. Mill unterstreicht die peremptorische Dringlichkeit dieser institutionell bedingten Pflichten, wenn er sagt, dass die Gesellschaft berechtigt sei, „diese Bedingungen um jeden Preis gegenüber jenen durchzusetzen, die sich anschicken, ihre Erfüllung zu verweigern“ (CW XVIII, S. 276; meine Hervorh.). Wie schon die vitalen Schutzpflichten des ersten Typs sollten daher auch die institutionellen Pflichten des zweiten Typs positiviert und mit Zwang bewehrt werden.

Worin unterscheiden sich die vollkommenen institutionellen Pflichten des zweiten Typs von den vollkommenen vitalen Schutzpflichten des ersten Typs? Warum betrachtet Mill die Pflichten zur Sicherung der Funktionstüchtigkeit gesellschaftlicher Institutionen des zweiten Typs überhaupt als Pflichten eigener Art? Einen möglichen Grund hatte ich schon genannt: Während vitale Schutzpflichten des ersten Pflichttyps direkt aus der moralischen Notwendigkeit der Sicherung vitaler Interessen erwachsen, entstehen die institutionellen Pflichten des zweiten Typs erst im Zuge von Institutionalisierungen. Beispielsweise setzt die institutionelle Pflicht, vor Gericht als Zeuge auszusagen, die Existenz eines Justizwesens voraus. Die Zeugnispflicht findet ihre unmittelbare Rechtfertigung zunächst in den unmittelbaren Bedürfnissen dieser Institution. Insofern jedoch ein reibungslos funktionierendes Justizwesen den vitalen Interessen aller dient, ist die institutionelle Zeugnispflicht in einem gewissen Sinne auch eine vitale Schutzpflicht - wenn auch nur in einem indirekten, institutionell vermittelten Sinne.

Ein weiterer Grund, der Mill dazu bewogen haben könnte, institutionelle Pflichten als Pflichten sui generis zu betrachten, ist dieser. Nach Mill kommt das absichtliche Wecken von Erwartungen bei anderen Menschen oft einem impliziten Versprechen gleich, das zu brechen ungerecht ist, weil es jemandes Recht verletzt: Es ist „ungerecht, jemandem die Treue zu brechen: eine ausdrückliche oder implizite Übereinkunft [engagement] nicht einzuhalten oder Erwartungen zu enttäuschen, die wir durch unser eigenes Verhalten geweckt haben, zumindest, wenn wir sie wissentlich geweckt haben“ (CW X, S. 242 f.; Hervorh. i. Orig.). Schon der passive Genuss der Annehmlichkeiten, die ein Leben im Schutz der Gesellschaft bietet, so Mill, weckt in unseren Mitmenschen legitime Erwartungen, die 
Rechte für sie und korrelative Pflichten für uns generieren: In „dem Maße, in dem jeder, der von den Vorteilen einer Gesellschaft profitiert, andere dazu veranlasst, von ihm all die guten Dienste und selbstlosen Leistungen zu erwarten, welche der moralische Fortschritt der Menschheit gebräuchlich gemacht hat, verdient er moralische Vorwürfe, wenn er, ohne guten Grund, diese Erwartung enttäuscht“ (Mill 1865a, S. 338).

Das dynamische, pflichtengenerierende „Prinzip“, auf das Mill hier anspielt, ähnelt einem Gerechtigkeitsgrundsatz, den H. L. A. Hart berühmt gemacht hat, und der heute meist „Fairnessprinzip“ genannt wird. Hart formuliert ihn so: „Wenn eine Anzahl von Personen irgendein gemeinsames Vorhaben nach Regeln durchführt und folglich ihre Freiheit einschränkt, haben diejenigen, die sich diesen Beschränkungen nötigenfalls unterworfen haben, ein Recht [a right] auf eine ähnliche Unterwerfung seitens derer, die von ihrer Unterwerfung begünstigt wurden. “7 Demnach hat jeder, der sich zur Realisierung ,gemeinschaftlicher Vorhaben im Interesse der Gesellschaft“ (CW XVIII, S. 225) freiwillig Regeln unterwirft, ein moralisches Recht darauf, dass andere es ihm gleichtun. Es liegt nahe, die durch das Fairnessprinzip generierten korrelativen Pflichten als „Fairnesspflichten“ zu bezeichnen.

Sollte es sich bei Mills Pflichten des zweiten Typs um Hartsche Fairnesspflichten handelt, dann würde ein signifikanter Unterschied zu den vitalen Schutzpflichten des ersten Typs deutlich, der ihre Klassifikation als Pflichten eigener Art rechtfertigen würde. Auf die Erfüllung vitaler Schutzpflichten haben alle Menschen ein Recht, weil sie auf ihren natürlichen Interessen als selbstkultivierende Wesen beruhen und von kontingenten Leistungen und Umständen unabhängig sind. Vitale Schutzpflichten sind universal und statisch, weil sie in den „dauerhaften“ Interessen aller Menschen fundiert sind. Hingegen haben wir Fairnesspflichten nur gegenüber jenen, die durch ihren fairen Beitrag zur Funktionstüchtigkeit gesellschaftlicher Institutionen ein Recht auf ihre Erfüllung erworben haben. Fairnesspflichten sind partikular und dynamisch, denn sie entstehen und vergehen mit den legitimen Erwartungen, die wir in unseren Mitmenschen durch unser fortgesetztes Tun und Lassen wecken: „Aufgrund dieses Prinzips erweitert sich der Bereich der moralischen Pflichten in einer sich verbessernden Gesellschaft ständig“ (Mill 1865a, S. 338).

Wir können an dieser Stelle offen lassen, wie plausibel das so verstandene Fairnessprinzip ist. Klar ist, dass die Gesellschaft in Mills Augen das moralische Recht hat, ihren Mitgliedern zwei Typen positiv-rechtlicher und positiv-morali-

7 Hart 1955, S. 185. - Die heute geläufigen Bezeichnungen „Fairnessprinzip“ oder „Grundsatz des Fair Play“ scheint Rawls geprägt zu haben. Vgl. Rawls 1964; 1971, Kapitel II, 18. 
scher Pflichten aufzuerlegen. Der erste Typ umfasst universale und weitgehend statische Pflichten zum Schutz vitaler und dauerhafter menschlicher Interessen. Hingegen besteht der zweite Typ aus Pflichten, die sich erst aus der Etablierung und Aufrechterhaltung von Institutionen zur Sicherung vitaler Pflichten des ersten Typs ergeben. Dieser Pflichttyp enthält institutionelle Fairnesspflichten, die vergleichsweise partikular und dynamisch sind ${ }^{8}$

Nach Mills Überzeugung ist die Gesellschaft nicht nur zur Positivierung und Durchsetzung dieser zwei Pflichttypen befugt. Mill erkennt in Kapitel IV von Über die Freiheit einen dritten Pflichttyp an, dessen Instanzen nur durch positivmoralische, aber nicht durch positiv-rechtliche Maßnahmen erzwungen werden dürfen. Die Gesellschaft dürfe mit den Mitteln der positiven Moral Handlungen verbieten und bestrafen, die zwar niemandes Rechte verletzen, aber dennoch den Interessen einiger abträglich seien:

Die Handlungen eines Individuums können für andere schmerzlich sein, oder es an der gebotenen Rücksicht für ihr Wohl missen lassen, ohne so weit zu gehen, ihre etablierten Rechte [constituted rights] ${ }^{9} \mathrm{zu}$ verletzen. Der Missetäter kann dann berechtigt durch die [öffentliche] Meinung bestraft werden, aber nicht durch das [positive] Recht (CW XVIII, S. 276; meine Hervorh.).

Da diese (nur positiv-moralisch) strafbaren Rücksichtslosigkeiten niemandes Rechte verletzten, kann es sich nicht um Verletzungen vollkommener Pflichten handeln. Sind sie also unvollkommene Pflichten, für die charakteristisch ist, dass „die speziellen Anlässe zu ihrer Erfüllung in unserem Ermessen liegen“ (CW X, S. 247)? Schwerlich, denn die Anlässe rücksichtsvollen Verhaltens sind nicht in unser Ermessen gestellt. Gewiss sollten wir immer gegenüber allen unseren Mitmenschen rücksichtsvoll zu sein, die unser Verhalten tangiert. Wie es scheint, haben wir es hier mit einer dritten Klasse moralischer Pflichten zu tun, die weder

8 Wie weit die Ähnlichkeiten zwischen Mills institutionellen Pflichten und Harts Fairnesspflichten reichen, muss hier offen bleiben. Harts Fairnessprinzip ist kontrovers, und auch Mills Assimilation des wissentlichen Weckens von Erwartungen an Versprechen ist mit guten Gründen kritisiert worden. Siehe z. B. Lyons 1994, S. 84.

9 Apropos „constituted rights.“ Mill schreibt: „The acts of an individual may be hurtful to others, or wanting in due consideration for their welfare, without going the length of violating any of their constituted rights.“ Der Kontext macht klar, dass Mill mit „constituted rights“ jene durch Regeln der Gerechtigkeit etablierten Rechte meint, von denen er fünf Zeilen zuvor sagt, dass sie als positive Rechte anzuerkennen seien. „Constituted rights“ sind also weder Verfassungsrechte noch überhaupt positive Rechte, sondern durch Regeln der Gerechtigkeit konstituierte moralische Rechte, die positiviert - „anerkannt“ - werden sollten, aber es nicht notwendig sind. 
vollkommen noch unvollkommen sind.$^{10}$ Ihre Übertretung ist ein „gesellschaftliches Vergehen“ (CW XVIII, S. 282), das mit Verstößen gegen unvollkommene Pflichten gemein hat, dass dadurch keine Rechte verletzt werden. Aber wie bei den vollkommenen Pflichten können wir uns das Wann, Wo und Wie ihrer Erfüllung nicht aussuchen.

„Wer es an der Rücksicht mangeln lässt, die allgemein den Interessen und Gefühlen anderer geschuldet ist ... ist Gegenstand moralischer Missbilligung für diese Verfehlung “ (CW XVIII, S. 281). Was wären Beispiele für moralisch strafwürdige Rücksichtslosigkeiten dieser Art? Nach Mill ist in diesem Sinne rücksichtslos, wer z. B. gewohnheitsmäßig gegenüber seinen Mitmenschen unfreundlich oder undankbar ist. Dasselbe gilt für die „Kultivierung von Angewohnheiten, die für sich genommen nicht lasterhaft sind“, für die der Einzelne jedoch bestraft werden darf, „wenn sie schmerzhaft sind für die, mit denen er sein Leben verbringt, oder für die, die aufgrund persönlicher Bindungen in ihrem Wohl von ihm abhängen“ (CW XVIII, S. 281). Ähnliches dürfte für „Verstöße gegen den Anstand“ gelten, die Mill so beschreibt: „Es gibt viele Handlungen, die [...] nicht rechtlich verboten werden sollten, die jedoch, wenn sie öffentlich getan werden, eine Verletzung guter Umgangsformen darstellen, und die daher, als zur Kategorie der Vergehen gegen andere gehörig, mit Recht bestraft werden dürfen. Von dieser Art sind Verstöße gegen den Anstand“ (CW XVIII, S. 295). Möglicherweise denkt Mill bei dieser Kategorie an das öffentliche Gebaren von Exzentrikern wie Diogenes von Sinope (†323 v. u. Z.), von dem berichtet wird, er habe zum großen Verdruss seiner Athener Mitbürger in aller Öffentlichkeit auf dem Marktplatz masturbiert, uriniert und seine Notdurft verrichtet. Es liegt nahe, diese Klasse legitimer gesellschaftlicher Forderungen als „Pflichten der Rücksichtnahme“ zu bezeichnen. Allerdings dürfen Rücksichtslosigkeiten dieser Art in Mills Augen nicht kriminalisiert, sondern nur durch positiv-moralischen Druck erzwungen werden dürfen. Denn ihre Durchsetzung dient nicht der (direkten oder indirekten) Sicherung vitaler Interessen, sondern primär der Aufrechterhaltung und Kultivierung eines angenehmen gesellschaftlichen Milieus.

Mit der Auflistung und Erläuterung dieser drei Typen von Pflichten beschließt Mill die inhaltliche Bestimmung des gesellschaftlichen Kontrollrechts. Diese und nur diese drei Typen von Pflichten darf die Gesellschaft ihren Mitgliedern moralisch gerechtfertigt auferlegen. Wir können die provisorische Formulierung des

10 Das ist insofern problematisch, als Mills Erläuterungen des naturrechtlichen Pflichtendualismus' in Kap. V von Utilitarismus den Eindruck erwecken, dass er in seinen Augen den Bereich des Moralischen erschöpft, dass also alle moralischen Pflichten entweder vollkommene oder unvollkommene sind: CW X, S. 247 f. 
Freiheitsprinzips aus Kapitel I von Über die Freiheit nun durch diese deontisch konkretisierte Fassung ersetzen. Der „einzige Zweck“, so Mills ursprüngliche Formulierung in Kapitel I, „zu dem Macht mit Recht über ein Mitglied einer zivilisierten Gemeinschaft gegen seinen Willen ausgeübt werden darf, [besteht] in der Verhinderung einer Schädigung anderer" (CW XVIII, S. 223; meine Hervorh.). Durch Ersetzung des vagen Schädigungsbegriffs durch Mills dreifache Unterscheidung zwischen natürlichen Pflichten, Fairnesspflichten und Pflichten der Rücksichtnahme und deren jeweilige Verletzung erhalten wir Mills deontisch konkretisiertes Freiheitsprinzip:

Der einzige Zweck, zu dem normative Autorität mit Recht über ein Mitglied einer zivilisierten Gemeinschaft gegen seinen Willen ausgeübt werden darf, besteht in der (positiv-rechtlichen bzw. positiv-moralischen) Setzung und Durchsetzung von vitalen Pflichten, Fairnesspflichten und Pflichten der Rücksichtnahme.

Diese drei Typen von Pflichten erschöpfen das, was Mill als die Sphäre „gesellschaftlicher Moralität, als Pflicht gegenüber anderen“ (CW XVIII, S. 283) bezeichnet. Ich werde daher im Folgenden die von Mill diskutierten drei Typen von Pflichten summarisch als „die gesellschaftlichen Pflichten“ bezeichnen.

\subsection{Drei Einwände gegen das konkretisierte Freiheitsprinzip}

Demnach besteht bürgerliche Freiheit in einer moralischen Immunität gegen die gesellschaftliche Auferlegung und zwangsweise Durchsetzung von Pflichten, die weder vitale Pflichten noch Fairnesspflichten oder Pflichten der Rücksichtnahme sind. Mit dieser Formulierung des konkretisierten Freiheitsprinzips in Kapitel IV beschließt Mill das Hauptargument von Über die Freiheit. Der Rest von Kapitel IV ist der Verteidigung und Erläuterung dieser Auffassung von Autorität und Freiheit gewidmet. Zunächst formuliert und diskutiert Mill der Sache nach drei mögliche Einwände gegen „diese Lehre“ (CW XVIII, S. 276). Betrachten wir sie der Reihe nach.

\section{Der Vorwurf „,selbstsüchtiger Gleichgültigkeit“ gegenüber anderen}

Der erste Vorwurf lautet, dass Mills konkretisiertes Freiheitsprinzip implizit zu einer Einstellung „selbstsüchtiger Gleichgültigkeit“ gegenüber dem Wohl anderer auffordere, weil es Zwang nur gegen Handlungen vorsieht, die unser Eigeninte- 
resse gefährden. Ist damit nicht gesagt, dass die Mitglieder einer Gesellschaft „sich um das Wohlergehen und Wohlsein anderer solange nicht kümmern sollten als ihre eigenen Interessen nicht berührt sind“? (CW XVIII, S. 276f.) Bedeutet dies nicht, dass die Gesellschaft kein Recht hat, in die Lebensführung ihrer Bürger einzugreifen, solange sie durch ihr Treiben ausschließlich ihr Eigenwohl beeinträchtigen?

Mill beeilt sich zu versichern, dass diese Interpretation des konkretisierten Freiheitsprinzips ein „grobes Missverständnis“ wäre. Die Gesellschaft habe sehr wohl ein Recht, auch dem rein selbstschädigenden Verhalten ihrer Mitglieder entgegenzutreten und zu diesem Zweck in ihre private Lebensführung einzugreifen. Er fordert sogar, dass die Gesellschaft von diesem Recht weit mehr Gebrauch machen sollte, als sie es - selbst im viktorianischen England seiner Zeit! - de facto tut: „Anstelle einer Verminderung bedarf es einer erheblichen Steigerung desinteressierter Anstrengungen zum Wohl anderer“ (CW XVIII, S. 277). Die Gesellschaft sollte vermehrt eingreifen, weil die rein selbstbezüglichen Tugenden für das Gemeinwohl (fast) ebenso bedeutsam seien wie die, die sich in der habituellen Erfüllung gesellschaftlicher Pflichten manifestiert: „Ich bin der Letzte, der die selbstbezüglichen Tugenden unterschätzt: Hinsichtlich ihrer Bedeutung sind sie nur zweitrangig, wenn sie es denn sind, gegenüber den gesellschaftlichen“ (CW XVIII, S. 277). Aus diesem Grund schulden wir einander Unterstützung auch bei der Realisierung unseres rein privaten Glücks: „Die Menschen schulden einander Hilfe bei der Unterscheidung des Besseren vom Schlechteren und Ermutigung, Ersteres zu wählen und Letzteres zu meiden. Sie sollten einander fortwährend anregen zur gesteigerten Ausübung ihrer höheren Fähigkeiten und zur gesteigerten Ausrichtung ihrer Gefühle und Zwecke auf weise anstatt auf törichte, auf erhebende anstatt auf erniedrigende Gegenstände und Betrachtungen“ (CW XVIII, S. 277).

Worin also besteht das „grobe Missverständnis“ des konkretisierten Freiheitsprinzips, vor dem Mill warnt? Es besteht darin, es als ein moralisches Prinzip aufzufassen, das wohlmeinende Eingriffe in die private Lebensführung auch mündiger Mitbürger reifen Alters tout court untersagt. In Wahrheit fordert das konkretisierte Freiheitsprinzip keinerlei Abstriche beim Ziel der allgemeinen Glücksbeförderung. Es beschneidet lediglich die Wahl der dafür zulässigen Mittel. Da es nur beansprucht, den Umgang der Gesellschaft mit dem Individuum durch Zwang und Kontrolle zu regeln, beschränkt es allein das gesellschaftliche Kontrollrecht, Befehle zu geben und Gehorsam zu erzwingen. Grundsätzlich ist das konkretisierte Freiheitsprinzip sogar mit einem beliebigen Maß zwangloser, rein beratender Einmischung in alle Lebensbereiche anderer Menschen verträglich, einschließlich ihres Privatlebens. Denn, so Mill, „desinteressiertes Wohlwollen kann andere Instrumente finden, die Leute $\mathrm{zu}$ ihrem eigenen Wohl zu überreden, 
als Peitschen und Knuten, der buchstäblichen oder der metaphorischen Sorte“ (CW XVIII, S. 277). Einem sich in Ratschlägen, Überredungsversuchen, Warnungen, Bitten und Flehen u. dgl. artikulierendem desinteressierten Wohlwollen sind in Mills Augen keine moralischen Grenzen gesetzt - solange ihr Adressat das letzte Wort behält: „Hilfe bei der Urteilsfindung, Ermahnungen zur Willensstärkung mögen [einer Person] von anderen angeboten, sogar aufgedrängt werden; aber sie bleibt der letzte Richter" (CW XVIII, S. 277; meine Hervorh.). Entscheidend ist, ob das „desinteressierte Wohlwollen“ der Gesellschaft sich in Befehlen und Drohungen artikuliert oder in bloßen Ratschlägen, deren Ablehnung ungestraft bleibt. Denn die Befolgung eines Rats ist dem Adressaten freigestellt, während einem autoritativen Befehl gehorcht werden muss. Salopp gesprochen sind Befehle Angebote, die man nicht ablehnen kann, während es zum Wesen eines Rats gehört, dass er straflos zurückgewiesen werden kann und darf. ${ }^{11}$

\section{Die „großartige Pflicht zur Selbstkultivierung“}

Mills Klarstellung provoziert einen neuen, diesmal gegen die Absolutheit des konkretisierten Freiheitsprinzips gerichteten Vorbehalt. Gewiss, so ließe sich einwenden, kann desinteressiertes Wohlwollen oft andere Mittel zur „Einschärfung“ [inculcation] der selbstbezüglichen Tugenden finden als Peitsche und Knute. Aber vielleicht nicht immer? Will Mill etwa sagen, dass es moralisch gefordert sei, diejenigen ihrem traurigen Schicksal zu überlassen, die unseren wohlmeinenden Rat ignorieren und an ihren selbstzerstörerischen Lebensweisen festhalten? Mit anderen, nämlich Mills eigenen Worten: „Sollte die Gesellschaft diejenigen ihrer eigenen Lebensführung überlassen, die dazu offenkundig unfähig sind? Wenn Glücksspiel oder Trunkenheit oder Hemmungslosigkeit oder Faulheit

11 Mill rekurriert hier auf die alte Unterscheidung zwischen Befehl und Rat, die Hobbes so expliziert: „Um daher richtig zu verstehen, was es heiße Befehlen, Ratgeben und Zureden, so muß die Definition dieser Wörter so bestimmt werden: Befehlen ist, wenn jemand zu demjenigen sagt, welcher weiß, daß der Grund von dem, was er tun soll, im Willen dessen, der redet, besteht: tue das oder tue das nicht; folglich muß der Befehlende seinen eigenen Vorteil zur Absicht haben, weil er verlangt, daß sein Wille als hinreichender Grund angenommen werden soll. Und will jemand etwas, so setzt dies allemal voraus, daß er darunter seinen Vorteil sucht. - Rat gibt man alsdann, wenn man zwar auch sagt: tue das oder tue es nicht; aber zur Ursache dabei hat, daß es demjenigen, zu welchem man es sagt, zum Vorteil gereiche. Der Ratgeber sucht folglich das Beste des andern“ (Leviathan, Kap. XXV: Vom Ratgeben; Hervorh. i. Orig.) - Natürlich behaupte ich nicht, dass Mill sich Hobbes' Explikation dieser Unterscheidung zu eigen macht. 
oder Unreinlichkeit ${ }^{12}$ für das Glück und die Verbesserung [eines Menschen] ein ebenso großes Hindernis bilden wie die meisten rechtlich verbotenen Handlungen, warum sollte (so mag man fragen) nicht das Recht bemüht sein, so weit es durchführbar und gesellschaftlich nützlich ist, auch sie zu unterdrücken? Und sollte nicht, in Ergänzung der unvermeidlichen Unvollkommenheiten des Rechts, zumindest die [öffentliche] Meinung eine mächtige Polizei gegen diese Laster bilden und streng mit gesellschaftlichen Strafen diejenigen heimsuchen, die sie praktizieren?“ (CW XVIII, S. 280 f.) Wären diesen Fragen zu bejahen, dann könnte das im Freiheitsprinzip artikulierte Verbot positiv-rechtlicher und positiv-moralischer Zwangsmittel für die gesellschaftliche Einschärfung auch selbstbezüglicher Tugenden zumindest nicht in der von Mill geforderten Absolutheit gelten.

Wahrscheinlich denkt Mill bei diesem zweiten Einwand gegen das konkretisierte Freiheitsprinzip an seinen Lehrer Jeremy Bentham. ${ }^{13}$ Bentham hätte ein Verbot gesellschaftlichen Zwangs für den Privatbereich, wie Mills konkretisiertes Freiheitsprinzip es vorsieht, abgelehnt. Denn in Benthams Augen bildet die Erfüllung auch rein selbstbezüglicher Pflichten einen zentralen Bestandteil der Ethik:

\begin{abstract}
Was die Ethik im Allgemeinen angeht, hängt das Glück eines Menschen in erster Linie von den Anteilen seines Verhaltens ab, an denen niemand anderes als er selbst interessiert ist; an zweiter Stelle von denjenigen Anteilen, die das Glück seiner Mitmenschen beeinflussen. In dem Maße, indem sein Glück von den erstgenannten Verhaltensanteilen abhängt, sagt man, dass es von seiner Pflicht gegenüber sich selbst abhängt. Folglich kann die Ethik als Kunst der Anleitung menschlichen Verhaltens in dieser Hinsicht als die Kunst der Erfüllung unsrer Pflicht uns selbst gegenüber bezeichnet werden: und die Qualität, die ein Mensch mit der Erfüllung dieser Art von Pflicht [...] beweist, ist die der Klugheit. ${ }^{14}$
\end{abstract}

12 „Unreinlichkeit“ [uncleanleness] im hier einschlägigen Sinne hat nichts mit Duschfrequenz und Seifenverbrauch zu tun, sondern ist ein viktorianischer Euphemismus für Promiskuität.

13 Aber vielleicht nicht nur, denn Mill antizipiert damit auch die Reaktion seines (ehemaligen) Freundes Thomas Carlyle. In einem Brief vom 04. Mai 1859 berichtet Carlyle seinem Bruder John von seiner ersten Lektüre von Über die Freiheit: „Habe ich Dir nicht Mills Essay Freiheit geschickt? [...] In meinem ganzen Leben habe ich noch nie einen ernsten geistreichen klaren logischen Essay mit vollkommener und tieferer Ablehnung seiner Grundlagen und der Mehrzahl seiner Schlussfolgerungen gelesen. [...] Als ob es Sünde wäre, menschliches Schwein in jeder Form zu kontrollieren oder zu besseren Weisen zu zwingen [...] Ach Gott im Himmel!“ (Meine Hervorh.; zit. n. http://carlyleletters.dukejournals.org, TC-JAC, 4 May. MS: NLS 516.69. Quot: A. Carlyle, NL 2:196. - Carlyles abschließender Stoßseufzer: „Ach Gott im Himmel!“ ist im Original auf Deutsch.

14 Bentham 1789, Kap. XVII, §6; Hervorh. i. Orig. - In Kapitel XVI bezeichnet Bentham den Verstoß gegen eine selbstbezügliche Pflicht als ein „selbstbezügliches Delikt“ [a self-regarding offence], das prinzipiell ebenso strafbar ist wie die Verletzung fremdbezüglicher Pflichten (Bentham 1789, Kap. XVI, §15). 
Mills Frage, ob nicht - „soweit es durchführbar und gesellschaftlich nützlich ist“ - selbstschädigendes Verhalten mitunter sogar mit positiv-rechtlichen Mitteln unterdrückt werden sollte, wird von Bentham ausdrücklich bejaht. Zwar gebe es, so Bentham, nur „wenige Fälle, in denen es zweckmäßig wäre, eine Menschen dafür zu bestrafen, dass er sich selbst verletzt“ (Bentham 1789, Kap. XVII, §18; Hervorh. i. Orig.). Aber grundsätzlich dürften, so Bentham, auch „Delikte gegen das eigene Selbst“ bestraft und die Erfüllung von „Pflichten gegenüber dem eigenen Selbst“ von der Gesellschaft rechtlich erzwungen werden.

In Mills Augen beweist Bentham mit dieser Haltung ein tiefes und folgenreiches Missverständnis der „großartigen Pflicht der Selbstkultivierung“. In einer zwei Jahrzehnte vor Über die Freiheit erschienenen Kritik an Benthams Moralphilosophie wirft Mill seinem Lehrer in scharfem Ton vor, die moralische Pflicht zur Selbstkultivierung ignoriert zu haben:

\footnotetext{
Es ist unnötig, sich über die Mängel eines Systems der Ethik zu verbreiten, das nicht beansprucht, Individuen bei der Charakterbildung zu unterstützen; das einen Wunsch nach Selbstkultivierung nicht anerkennt, ja nicht einmal ein Vermögen dazu als in der menschlichen Natur existierend; und das, selbst wenn es ihn anerkennen würde, nur wenig Hilfestellung bei der Erfüllung dieser großartigen Pflicht leisten könnte [...] Die Moral [morality] besteht aus zwei Teilen. Eines von ihnen ist Selbsterziehung; die Schulung, durch den Menschen selbst, seiner Affekte und seines Willens. Dieser Teil bleibt leer in Benthams System (Mill 1838, S. 98).
}

Auf den ersten Blick erscheint Mills Vorwurf, dass Selbstkultivierung in Benthams Ethik keine Rolle spiele, überraschend: Haben wir nicht gerade gesehen (s. das Zitat oben), dass Bentham selbstbezügliche Pflichten explizit anerkennt und einen Teilbereich der Ethik geradezu als „Kunst der Erfüllung unserer Pflicht uns selbst gegenüber“ bezeichnet? In der Tat. Aber Mills Kritik richtet nicht ihr Fehlen, sondern gegen Benthams angebliche Verkennung der überragenden Bedeutung dieser „großartigen Pflicht“ (s. o.) für menschliche Selbstkultivierung. Mill bemängelt, dass das, was Bentham als „selbstbezügliche Pflichten“ bezeichnet, nichts mit tugendhafter Charakterbildung und Selbstkultivierung zu tun habe. Denn für Bentham erschöpfen sich selbstbezügliche Pflichten in bloßen Geboten der Klugheit: Man sollte eben keine Dummheiten begehen, die dem eigenen Glück abträglich sind. Für Mill hingegen gehen selbstbezügliche Pflichten weit über die bloße Vermeidung von Dummheiten hinaus. Ihr Gegenstand ist die aktive Verbesserung des eigenen Selbst: die Erziehung, Bildung und Kultivierung der eigenen Persönlichkeit gemäß einem „Ideal der Vervollkommnung der menschlichen Natur“ (CW XVIII, S. 278). Denn die möglichst weitgehende Verwirklichung dieses ,griechischen Ideals der Selbstentwicklung“ (CW XVIII, S. 266) bildet für Mill einen der „Hauptbestandteile menschlichen Glücks 
und durchaus den Hauptbestandteil individuellen und gesellschaftlichen Fortschritts. “15

In Kapitel IV von Über die Freiheit kommt Mill auf diesen Punkt zurück. Contra Bentham betont er dort erstens, dass selbstbezügliche Pflichten ,nicht gesellschaftlich verpflichtend“ seien und daher von der Gesellschaft nicht erzwungen werden dürften; und zweitens, dass die Klasse der selbstbezüglichen Pflichten neben Klugheitspflichten mindestens auch - und vor allem - die „großartige“ Pflicht zur Selbstkultivierung umfasst:

Was Pflichten uns selbst gegenüber genannt wird, ist nicht gesellschaftlich verpflichtend, es sei denn, die Umstände machen sie zugleich zu Pflichten gegenüber anderen. Der Term Pflicht gegenüber sich selbst, wenn damit mehr als Klugheit gemeint ist, bedeutet Selbstrespekt oder Selbstentwicklung: und für keines von diesen ist man seinen Mitmenschen verantwortlich, weil es für keines von ihnen zum Wohl der Menschheit ist, dass man verantwortlich gemacht wird. ${ }^{16}$

Von entscheidender Bedeutung ist hier, dass „Selbstkultivierung“ für Mill nicht nur Kultivierung des eigenen Selbst meint, sondern vor allem Kultivierung durch das eigene Selbst. Sie ist wesentlich „Selbsterziehung; die Schulung, durch den Menschen selbst, seiner Affekte und seines Willens“ (Mill 1838, S. 98; meine

15 CW XVIII, S. 261; vgl. den Beitrag von Schramme, im vorliegenden Band.

16 CW XVIII, S. 279. - Mills distanzierende Formulierung ,was Pflichten gegenüber uns selbst genannt wird“ wird oft als Beleg dafür angeführt, dass Mill in Über die Freiheit nicht mehr bereit ist, selbstbezügliche Pflichten als genuin moralische Pflichten anzuerkennen. Angesichts der unzweifelhaften Bedeutung, die Mill der Sache nach der „großartigen Pflicht zu Selbstkultivierung“ auch in Über die Freiheit beimisst, scheint jedoch klar, dass Mills Unbehagen rein terminologischer Natur ist. Er hält es zur Zeit der Abfassung von Über die Freiheit für zweckmäßiger, den Pflichtbegriff mit dem Bereich des „gesellschaftlich Verbindlichen“ zu identifizieren. Während Mill in seiner Benthamkritik von 1838 es - Bentham folgend - noch für zweckmäßiger hielt, den Bereich der Moral in einen fremd- und einen selbstbezüglichen Bereich aufzuteilen (s. das Zitat oben), hält er es nun - um 1859 - für angemessener, innerhalb des übergreifenden Gebiets der „Lebenskunst“ [art of living] das Teilgebiet der „Moral“ zu unterscheiden und allein die „gesellschaftlich verbindlichen“ Forderungen der Lebenskunst als „Pflichten“ zu bezeichnen. Wie wir sahen, geht diese terminologische Verschiebung für Mill nicht einher mit einer inhaltlichen Abwertung dessen, was Bentham und Mills früheres Selbst „Pflichten gegenüber sich selbst“ genannt haben. Das zeigt sich auch daran, dass Mill wenige Jahre nach der Veröffentlichung von Über die Freiheit sich erneut der Terminologie von „Moral“ und „Pflicht“ bedient, wenn er einmal mehr die überragenden Bedeutung der Selbstkultivierung für Menschen als entwicklungsfähige Wesen betont: „Wir haben eine moralische Verpflichtung, die Verbesserung unseres Charakters zu erstreben“ (Mill 1865b, 466; meine Hervorh.; vgl. a. Mill 1874, S. 397: „[D]ie Pflicht des Menschen ist hinsichtlich seiner eigenen Natur dieselbe wie hinsichtlich der Natur aller anderen Dinge, nämlich nicht, ihr zu folgen, sondern sie zu verbessern“ [Hervorh. i. Orig.]. 
Hervorh.). Was Mill in der oben zitierten Benthamkritik als ,großartige Pflicht zur Selbstkultivierung“ bezeichnet, ist in einem doppelten Sinne selbstbezüglich: Nicht nur ist ihr Gegenstand das eigene Selbst, sondern dem Selbst allein obliegt ihre Erfüllung in exklusiver, souveräner Eigenverantwortlichkeit. Eben deshalb hat die Gesellschaft in Mills Augen kein Recht, die Pflicht zur Selbstkultivierung des Einzelnen zu erzwingen. Aber gehört es Mill zufolge nicht „zur Vorstellung der Pflicht in jeder ihrer Formen, dass eine Person berechtigt gezwungen werden darf, sie zu erfüllen“ (CW X, S. 246; meine Hervorh.)? Wenn das Freiheitsprinzip der Gesellschaft die Durchsetzung selbstbezüglicher Pflichten untersagt, wer darf sie dann berechtigt erzwingen? Nur das Individuum selbst. Wenn es um Selbstkultivierung geht, ist allein der Selbstzwang durch die „inneren Sanktionen“ des eigenen Gewissens legitim. Auch die für die Fortentwicklung der inneren Sanktionen nötige „Schulung“ der eigenen Affekte und des eigenen Willens geht in Mills Augen mit dem Erreichen der Mündigkeit vollständig auf das Individuum über und ist gesellschaftlichen Zwangsmaßnahmen fortan entzogen. ${ }^{17}$ Eben darin besteht für Mill die Hauptpointe des konkretisierten Freiheitsprinzips: Dass

17 Die unabsichtlichen „natürlichen Strafen“, die Mill in Kapitel IV erwähnt, sind in seinen Augen ebenso wenig echte Strafen sind wie Gummi-Enten echte Enten, und die mit ihnen verknüpfte rein kausale Nötigung ist kein wirkliches Zwingen im hier relevanten intentionalen Sinne. Wie Mill in Kapitel IV erläutert, kann es passieren, dass eine Person durch rein selbstbezügliches Verhalten de facto schwere Übel erleidet, obwohl sie keine der im konkretisierten Freiheitsprinzip genannten gesellschaftlichen Pflichten verletzt. Denn nach Mill ist es nicht nur möglich, sondern naturgesetzlich unvermeidlich, dass wir - nolens volens - anderen Menschen auch für ihre rein selbstbezüglichen Fehler schweres Leid zufügen: „Es gibt ein Ausmaß an Torheit und ein Ausmaß an [...] Niedrigkeit und Verderbtheit des Geschmacks, das, obwohl es keine Schädigung der Person, die es an den Tag legt, rechtfertigt, sie notwendig und zu Recht zu einem Gegenstand des Missfallens, oder, in Extremfällen, sogar der Verachtung machen [...] Obwohl sie niemandem Unrecht tut, kann eine Person sich so verhalten, dass sie uns nötigt [compel] sie urteilend und fühlend als Narren oder als Wesen niederen Ranges anzusehen [...] Auf diese verschiedenen Weisen kann eine Person sehr schwere Strafen durch die Hand anderer erleiden für Fehler, die direkt nur sie selbst betreffen; aber sie erleidet diese Strafen nur insoweit sie natürlich und sozusagen die unwillkürlichen Folgen der Fehler selbst sind, nicht weil sie ihnen absichtlich zum Zweck der Bestrafung zugefügt würden“ (CW XVIII, S. 278; meine Hervorh.). Diese „natürlichen Strafen“ sind keine genuinen Bestrafungen, eben weil sie nicht absichtlich zu diesem Zweck zugefügt werden. Bestenfalls sind sie Übel, die sich wie genuine Strafen anfühlen. Auch werden sie nur insofern „,von der Hand anderer erlitten“, als sie das letzte Glied einer Kausalkette bilden, zu deren Zwischengliedern die (nach Mill unvermeidliche, weil aufgrund psycho-physiologischer Gesetze erzwungene) kognitive und gefühlsmäßige Verurteilung des betreffenden Verhaltens durch andere Personen gehört. Die Kausalkette geht gewissermaßen durch die Psyche anderer Personen hindurch, aber sie wird dabei in ihrem Verlauf und in ihrer Wirkung von diesen Personen nicht willentlich kontrolliert. 
der Einzelne nicht nur eine Immunität, sondern darüber hinaus einen moralischen Anspruch darauf hat, für die Zwecke der Kultivierung seines Selbst durch sein Selbst von gesellschaftlichem Zwang verschont zu bleiben.

Fassen wir zusammen. Mill bekräftigt in seiner Replik auf den zweiten Einwand, dass wir einem Mitbürger, der unseren wohlmeinenden Rat konsequent ignoriert und an seinen selbstzerstörerischen Lebensweisen festhält, letztlich seinem traurigen Schicksal überlassen sollten. Zumindest gilt dies für einen mündigen Bürger reifen Alters: „Alle Fehler, die er entgegen allem Rat und aller Warnung wahrscheinlich begehen wird, werden bei Weitem aufgewogen durch das Übel einer Erlaubnis anderer, ihn zu dem zu zwingen [constrain], was sie als sein Wohl erachten“ (CW XVIII, S. 277). Die mit der erforderlichen Toleranz verbundenen sozialen Einbußen und Kosten, so Mill, muss eine freiheitliche Gesellschaft aushalten: Diese „Unannehmlichkeit ist eine, welche eine Gesellschaft um des größeren Guts der Freiheit Willen ertragen kann“ (CW XVIII, S. 282).

\section{Kein Mensch ist eine Insel}

Ein dritter von Mill diskutierter Einwand gegen das konkretisierte Freiheitsprinzip betrifft eine Distinktion, die Mill in Kapitel I von Über die Freiheit so erläutert: Es „gibt eine Handlungssphäre, an der die Gesellschaft, wenn überhaupt, im Unterschied zum Individuum nur ein indirektes Interesse hat; sie umfasst den gesamten Anteil des Lebens einer Person und ihres Verhaltens, der nur sie selbst betrifft, oder sofern sie auch andere betrifft, nur mit ihrem freien, willigen und ungetäuschten Einverständnis und ihrer Beteiligung. Wenn ich ,nur sie selbst' sage, dann meine ich direkt und in erster Linie: denn was immer sie betrifft, kann auch andere durch sie betreffen; und der Einwand, der auf dieser Möglichkeit beruht, wird in der Folge betrachtet werden“ (CW XVIII, S. 225). Mills Beschreibung der individuellen Freiheitssphäre suggeriert, dass alle Handlungen, die eine Person in ihrem Leben vollzieht, genau einer von zwei Klassen angehören: Selbstbezügliche Handlungen, deren Konsequenzen „direkt und in erster Linie“ nur ihre eigenen Interessen berühren; und solche Handlungen, die nicht selbstbezüglich sind, weil sie „direkt und in erster Linie“ - die Interessen anderer Personen tangieren (CW XVIII, S. 225). Die erste Klasse selbstbezüglicher Handlungen bildet die „Domäne der Freiheit“. Sie werden vom Freiheitsprinzip gegen gesellschaftlichen Zwang immunisiert. Die zweite Klasse bilden Handlungen, die gesellschaftliche Pflichten verletzen und für die das Freiheitsprinzip die Anwendung von Zwang und Kontrolle legitimiert. Diese zweite Klasse fremdbezüglicher Handlungen bilden die Pflichtsphäre der „Moralität und des Rechts“ (CW XVIII, S. 282; vgl. CW XVIII, S. 225; S. 276). 
Sein Versprechen aus Kapitel I, „in der Folge“ den diesbezüglichen Einwand zu diskutieren, löst Mill in Kapitel IV ein. Zunächst reformuliert er den Einwand: „Die hier aufgezeigte Unterscheidung zwischen dem Anteil des Lebens einer Person, der nur sie selbst und dem Anteil, der andere betrifft, werden viele Personen nicht einzuräumen bereit sein. Wie (so mag man fragen) kann ein irgendein Teil des Verhaltens eines Gesellschaftsmitglieds den anderen Mitgliedern gleichgültig sein? Kein Mensch ist ein isoliertes Wesen; es ist unmöglich, dass eine Person sich irgendeine ernstliche oder dauerhafte Verletzung zufügt, ohne dass Unheil [mischief] nicht zumindest die Nahbeziehungen erreicht und oft darüber hinausgeht“ (CW XVIII, S. 280; vgl. CW XVIII, S. 225). Kurz, der Vorwurf lautet, dass Mills Distinktion zwischen selbst- und fremdbezüglichen Handlungen de facto eine Unterscheidung ohne Unterschied ist, weil jede selbstbezügliche Handlung sich unweigerlich kausal auch auf andere Personen auswirkt und daher immer zugleich fremdbezüglich ist. Der Einwand wäre, sollte er triftig sein, für Mills Freiheitsprinzip fatal, weil die Klasse rein selbstbezüglicher Handlungen in diesem Fall leer wäre. Alles Tun und Lassen würde zur Pflichtsphäre gehören und wäre als solche gesellschaftlicher Kontrolle prinzipiell unterworfen. Eine befriedigende Replik auf diesen dritten Einwand muss zeigen, dass die Klasse der rein selbstbezüglichen Handlungen nicht leer ist und dass das konkretisierte Freiheitsprinzip ein praktikables Kriterium zu ihrer Identifikation bereitstellt.

In Kapitel I von Über die Freiheit formuliert Mill einen Kausaltest für Selbstbezüglichkeit: Eine Handlung betreffe „nur [die handelnde Person] selbst“, wenn ihre Primärfolgen direkt nur sie berühren und sich auf andere Personen bestenfalls durch sie, d. h. als Sekundärfolgen der Primärfolgen auf andere auswirke: „Wenn ich ,nur sie selbst' sage, dann meine ich direkt und in erster Linie: denn was immer sie betrifft, kann auch andere durch sie betreffen“ (CW XVIII, S. 225; meine Hervorh.). Wie mit Blick auf Mills konkretisiertes Freiheitsprinzip zu erwarten ist, wird dieser Kausaltest in Kapitel IV durch ein deontisches Kriterium in Begriffen gesellschaftlicher Pflichten ersetzt:

Ich gestehe vollkommen zu, dass Unheil, welches eine Person sich selbst zufügt, diejenigen, die mit ihr eng verbunden sind, und in geringerem Maße die Gesellschaft als Ganze, durch deren Sympathien und deren Interessen ernstlich beeinflussen kann. Wird durch ein Verhalten dieser Art eine Person dazu gebracht, eine distinkte und zuordenbare Pflicht [a distinct and assignable obligation] gegenüber irgendeiner anderen Person oder anderen Personen zu verletzen, fällt der Fall aus der selbstbezüglichen Klasse heraus und ist moralischer Missbilligung im eigentlichen Wortsinn ausgesetzt. ${ }^{18}$

18 CW XVIII, S. 281; meine Hervorh. - Abgesehen von der prinzipiellen Unklarheit der Unterscheidung zwischen Primär- und Sekundärfolgen ist das Kausalkriterium aus Kapitel I schon 
Es ist unklar, was genau ,distinkte und zuordenbare Pflichten gegenüber irgendeiner anderen Person oder anderen Personen“ sind. Aber Mills Beispiele scheinen zu bestätigen, dass er hier an die gesellschaftlichen Pflichten denkt, mit denen er wenige Seiten zuvor den Inhalt des Freiheitsprinzips konkretisiert hatte. Ist dies korrekt, dann können wir das von Mill angedeutete Identifikationskriterium für selbstbezügliche Handlungen so formulieren: Eine Handlung ist nicht selbstbezüglich und gehört der Pflichtensphäre an, wenn sie als Verletzung einer gesellschaftlichen Pflicht (re)formulierbar ist. Andernfalls ist sie selbstbezüglich und ein Element der Freiheitssphäre.

Betrachten wir zur Erläuterung abschließend die von Mill in Kapitel IV diskutierten Beispiele. Das ist zunächst der Mann, der durch Akte der „Verschwendung und Ausschweifung“ unfähig wird, seine Familie zu ernähren und seine Schulden zu bezahlen. Solange sein Tun nur verschwenderisch und ausschweifend ist, so Mill, ist es selbstbezüglich und freigestellt. Sobald seine Ausschweifungen ihn jedoch $\mathrm{zu}$ Verletzungen gesellschaftlicher Pflichten treiben oder sein Tun von vornherein als pflichtverletzend beschrieben werden kann, „wird er verdientermaßen verdammt und kann gerechtfertigt bestraft werden; aber für die Pflichtverletzung [...] nicht für die Ausschweifung“ (CW XVIII, S. 281; meine Hervorh.). Dasselbe gilt für schlechte, „für sich genommen nicht bösartige“ Angewohnheiten eines Menschen, die jedoch in dem Augenblick zu Verletzungen gesellschaftlicher Pflichten der Rücksichtnahme werden, wenn sie „schmerzhaft sind für die, mit denen er sein Leben verbringt, oder die aufgrund persönlicher Bindungen in ihrem Wohl von ihm abhängen“ (CW XVIII, S. 281). Jedoch macht auch ein solcher Mensch sich allein für die Pflichtverletzung, nicht für die Laster strafwürdig, die zu ihr geführt haben. Und schließlich: „Keine Person sollte für Trunkenheit bestraft werden; aber ein Soldat oder ein Polizist sollte bestraft werden für Trunkenheit im Dienst“ (OL, 282). Erneut kommt es auf die Beschreibung an: Trunkenheit ist für sich genommen kein legitimer Gegenstand gesellschaftlichen Zwangs, aber Trunkenheit-im-Dienst ist es. Entscheidend ist, ob die Handlung zur Verletzung einer gesellschaftlichen Pflicht führt oder - wie im letzten Fall - direkt als eine solche beschrieben werden kann.

deshalb unbrauchbar, weil Mill bestimmte Handlungen, die andere auch direkt, z. B. im ökonomischen Wettbewerb, kausal schädigen, ausdrücklich zulässt: CW XVIII, S. 293. - Ferner: Ist es überhaupt möglich, wie Mill behauptet, durch „Untätigkeit Übel zu verursachen“ (CW XVIII, S. 225)? Wie kann die Abwesenheit eines Tuns - ein Mangel, ein Nichts - ursächlich sein? Das ist das Problem der Kausalität von Unterlassungen. Aber selbst wenn wir annehmen, dass Abwesenheiten ursächlich sein können: Wie können wir bei ihnen zwischen Primär- und Sekundärwirkungen unterscheiden? 
Mills konkretisierende, auf den vagen Schädigungsbegriff verzichtende Reformulierung des Freiheitsprinzips in Begriffen gesellschaftlicher Pflichten in Kapitel IV ist sein letztes Wort in Über die Freiheit zur „praktischen Frage, wo die Grenze zu ziehen ist - wie der passende Abgleich zwischen individueller Freiheit und gesellschaftlicher Kontrolle vorzunehmen ist“ (CW XVIII, S. 220). Alles, was über die gesellschaftliche Durchsetzung vitaler Pflichten, Fairnesspflichten und Pflichten des Anstands hinausgeht, ist „usurpierte“, d. h. missbräuchlich angemaßte Autorität (CW XVIII, S. 283). Es ist jedoch zu beachten, dass missbrauchte Autorität für Mill ebenso wenig Autorität ist wie ein gefälschte Banknote eine Banknote. Fälschlich angemaßte gesellschaftliche Autorität zur Auferlegung von Pflichten ist keine besondere Form von Autorität, sondern deren Abwesenheit. Weil „usurpierte Autorität“ normativ wirkungslos ist - „null und nichtig“ - erzeugt sie keine Pflichten für den Bürger. In der Freiheitssphäre des Bürgers besitzt die Gesellschaft keine autoritative Setzungsbefugnis, und diese Nicht-Befugnis korreliert seitens des Bürgers mit einem moralischen Zustand der Immunität.

\section{Literatur}

Bain, Alexander (1882): John Stuart Mill. A Criticism with Personal Recollections. London: Longmans, Green \& Co.

Bentham, Jeremy (1907): An Introduction to the Principles of Morals and Legislation. 1789; zit. n. der Oxforder Ausgabe. Oxford: Clarendon Press.

Hart, H. L. A.(1955): „Are There Any Natural Rights?“.In: The Philosophical Review 64, 2, S. 175-191.

Hobbes, Thomas [1651] (2011): Leviathan, oder Stoff, Form und Gewalt eines kirchlichen oder und staatlichen Gemeinwesens. Berlin: Suhrkamp.

Hohfeld, Wesley N. (1913): „Some Fundamental Legal Conceptions as Applied in Legal Reasoning“. In: The Yale Law Journal 23, 1, S. 16-59; in deutscher Übersetzung in Stepanians (2007).

Lyons, David (1994): Rights, Welfare, and Mill's Moral Theory. New York [u. a.]: Oxford Univ. Press.

Rawls, John (1964): „Legal obligation and the duty of fair play“. In: Hook, S. (Hrsg.): Law and Philosophy: A Symposium. New York: New York University Press, S. 3-18.

Rawls, John (1971): A Theory of Justice. Cambridge, Mass: Belknap Press of Harvard Univ. Press. Stepanians, Markus (Hrsg.) (2007): Individuelle Rechte. Paderborn: Mentis. 